Georgian Mathematical Journal

1(94), No. 4, 367-376

\title{
TWO-WEIGHTED $L_{p}$-INEQUALITIES FOR SINGULAR INTEGRAL OPERATORS ON HEISENBERG GROUPS
}

\author{
V. S. GULIEV
}

\begin{abstract}
Some sufficient conditions are found for a pair of weight functions, providing the validity of two-weighted inequalities for singular integrals defined on Heisenberg groups.
\end{abstract}

Estimates for singular integrals of the Calderon-Zygmund type in various spaces (including weighted spaces and the anisotropic case) have attracted a great deal of attention on the part of researchers. In this paper we will deal with singular integral operators $T$ on the Heisenberg group $H^{n}$ which have an essentially different character as compared with operators of the Calderon-Zygmund type. We have obtained the two-weighted $L_{p^{-}}$ inequality with monotone weights for singular integral operators $T$ on $H^{n}$. Applications are given.

Let $H^{n}$ be the Heisenberg group (see [1], [2]) realized as a set of points $x=\left(x_{0}, x_{1}, \ldots, x_{2 n}\right)=\left(x_{0}, x^{\prime}\right) \in \mathbb{R}^{2 n+1}$ with the multiplication

$$
x y=\left(x_{0}+y_{0}+\frac{1}{2} \sum_{i=1}^{n}\left(x_{i} y_{n+i}-x_{n+i} y_{i}\right), \quad x^{\prime}+y^{\prime}\right) .
$$

The corresponding Lie algebra is generated by the left-invariant vector fields

$$
\begin{aligned}
X_{0} & =\frac{\partial}{\partial x_{0}}, \quad X_{i}=\frac{\partial}{\partial x_{i}}+\frac{1}{2} x_{n+i} \frac{\partial}{\partial x_{0}}, \\
X_{n+i} & =\frac{\partial}{\partial x_{n+i}}-\frac{1}{2} x_{i} \frac{\partial}{\partial x_{0}}, \quad i=1, \ldots, n,
\end{aligned}
$$

which satisfy the commutation relation

$$
\begin{gathered}
{\left[X_{i}, X_{n+i}\right]=\frac{1}{4} X_{0},} \\
{\left[X_{0}, X_{i}\right]=\left[X_{0}, X_{n+i}\right]=\left[X_{i}, X_{j}\right]=\left[X_{n+i}, X_{n+j}\right]=\left[X_{i}, X_{n+j}\right]=0,}
\end{gathered}
$$

1991 Mathematics Subject Classification. 42B20, 42B25, 42B30. 


$$
i, j=1, \ldots, n \quad i \neq j .
$$

The dilation $\delta_{t}: \delta_{t} x=\left(t^{2} x_{0}, t x^{\prime}\right), t>0$, is defined on $H^{n}$. The Haar measure on this group coincides with the Lebesgue measure $d x=d x_{0} d x_{1} \cdots d x_{2 n}$. The identity element in $H^{n}$ is $e=0 \in \mathbb{R}^{2 n+1}$, while the element $x^{-1}$ inverse to $x$ is $(-x)$.

The function $f$ defined in $H^{n}$ is said to be $H$-homogeneous of degree $m$, on $H^{n}$, if $f\left(\delta_{t} x\right)=t^{m} f(x), t>0$. We also define the norm on $H^{n}$

$$
|x|_{H}=\left[x_{0}^{2}+\left(\sum_{i=1}^{2 n} x_{i}^{2}\right)^{2}\right]^{1 / 4}
$$

which is $H$-homogeneous of degree one. This also yields the distance function, namely, the distance

$$
\begin{gathered}
d(x, y)=d\left(y^{-1} x, e\right)=\left|y^{-1} x\right|_{H} \\
\left|y^{-1} x\right|_{H}=\left[\left(x_{0}-y_{0}-\frac{1}{2} \sum_{i=1}^{n}\left(x_{i} y_{n+i}-x_{n+i} y_{i}\right)\right)^{2}+\right. \\
\left.+\left(\sum_{i=1}^{2 n}\left(x_{i}-y_{i}\right)^{2}\right)^{2}\right]^{1 / 4} .
\end{gathered}
$$

$d$ is left-invariant in the sense that $d(x, y)$ remains unchanged when $x$ and $y$ are both left-translated by some fixed vector in $H^{n}$. Furthermore, $d$ satisfies the triangle inequality $d(x, z) \leq d(x, y)+d(y, z), x, y, z \in H^{n}$. For $r>0$ and $x \in H^{n}$ let

$$
B(x, r)=\left\{y \in H^{n} ;\left|y^{-1} x\right|_{H}<r\right\} \quad\left(S(x, r)=\left\{y \in H^{n} ;\left|y^{-1} x\right|_{H}=r\right\}\right)
$$

be the $H$-ball ( $H$-sphere) with center $x$ and radius $r$.

The number $Q=2 n+2$ is called the homogeneous dimension of $H^{n}$. Clearly, $d\left(\delta_{t} x\right)=t^{Q} d x$.

Given functions $f(x)$ and $g(x)$ defined in $H^{n}$, the Heisenberg convolution ( $H$-convolution) is obtained by

$$
(f * g)(x)=\int_{H^{n}} f(y) g\left(y^{-1} x\right) d y=\int_{H^{n}} f\left(x y^{-1}\right) g(y) d y,
$$

where $d y$ is the Haar measure on $H^{n}$.

The kernel $K(x)$ admitting the estimate $|K(x)| \leq C|x|_{H}^{\alpha-Q}$ is summable in the neighborhood of $e$ for $\alpha>0$ and in that case $K * g$ is defined for the function $g$ with bounded support. If however the kernel $K(x)$ has a singularity of order $Q$ at zero, i.e., $|K(x)| \sim|x|_{H}^{-Q}$ near $e$, then there arises a singular integral on $H^{n}$. 
Let $\omega(x)$ be a positive measurable function on $H^{n}$. Denote by $L_{p}\left(H^{n}, \omega\right)$ a set of measurable functions $f(x), x \in H^{n}$, with the finite norm

$$
\|f\|_{L_{P}\left(H^{n}, \omega\right)}=\left(\int_{H^{n}}|f(x)|^{p} \omega(x) d x\right)^{1 / p}, \quad 1 \leq p<\infty .
$$

We say that a locally integrable function $\omega: H^{n} \rightarrow(0, \infty)$ satisfies Muckenhoupt's condition $A_{p}=A_{p}\left(H^{n}\right)$ (briefly, $\left.\omega \in A_{p}\right), 1<p<\infty$, if there is a constant $C=C(\omega, p)$ such that for any $H$-ball $B \subset H^{n}$

$$
\left(|B|^{-1} \int_{B} \omega(x) d x\right)\left(|B|^{-1} \int_{B} \omega^{1-p^{\prime}}(x) d x\right) \leq C, \quad \frac{1}{p}+\frac{1}{p^{\prime}}=1,
$$

where the second factor on the left is replaced by $\operatorname{ess} \sup \left\{\omega^{-1}(x): x \in B\right\}$ if $p=1$.

Let $K(x)$ be a singular kernel defined on $H^{n} \backslash\{e\}$ and satisfying the conditions: $K(x)$ is an $H$-homogeneous function of degree $-Q$, i.e., $K\left(\delta_{t} x\right)=$ $t^{-Q} K(x)$ for any $t>0$ and $\int_{S_{H}} K(x) d \sigma(x)=0$, where $d \sigma(x)$ is a measure element on $S_{H}=S(e, 1)$.

Denote by $\omega_{K}(\delta)$ the modulus of continuity of the kernel on $S_{H}$ :

$$
\omega_{K}(\delta)=\sup \left\{|K(x)-K(y)|: x, y \in S_{H}, \quad\left|y^{-1} x\right|_{H} \leq \delta\right\} .
$$

It is assumed that

$$
\int_{0}^{1} \omega_{K}(t) \frac{d t}{t}<\infty
$$

We consider the singular integral operator $T$ :

$$
T f(x)=\int_{H^{n}} K\left(x y^{-1}\right) f(y) d y=: \lim _{\varepsilon \rightarrow 0+} \int_{\left|x y^{-1}\right|_{H}>\varepsilon} K\left(x y^{-1}\right) f(y) d y .
$$

As is known, $T$ acts boundedly in $L_{p}\left(H^{n}\right), 1<p<\infty$ (see [3], [4]). For singular integrals with Cauchy-Szegö kernels the weighted estimates were established in the norms of $L_{p}\left(H^{n}, \omega\right)$ with weights $\omega$ satisfying the condition $A_{p}[5]$. These results extend to the more general kernels considered above [4].

Theorem 1 [4]. Let $1<p<\infty$ and $\omega \in A_{p}$; then $T$ is bounded in $L_{p}\left(H^{n}, \omega\right)$.

In the sequel we will use

Theorem 2. Let $1 \leq p \leq q<\infty$ and $U(t), V(t)$ be positive functions on $(0, \infty)$. 
1) The inequality

$$
\left(\int_{0}^{\infty} U(t)\left|\int_{0}^{t} \varphi(\tau) d \tau\right|^{q} d t\right)^{1 / q} \leq K_{1}\left(\int_{0}^{\infty}|\varphi(t)|^{p} v(t) d t\right)^{1 / p}
$$

with the constant $K_{1}$ not depending on $\varphi$ holds iff the condition

$$
\sup _{t>0}\left(\int_{t}^{\infty} U(\tau) d \tau\right)^{p / q}\left(\int_{0}^{t} V(\tau)^{1-p^{\prime}} d \tau\right)^{p-1}<\infty
$$

is fulfilled;

2) The inequality

$$
\left(\int_{0}^{\infty} U(t)\left|\int_{t}^{\infty} \varphi(\tau) d \tau\right|^{q} d t\right)^{1 / q} \leq K_{2}\left(\int_{0}^{\infty}|\varphi(t)|^{p} V(t) d t\right)^{1 / p}
$$

with the constant $K_{2}$ not depending on $\varphi$ holds iff the condition

$$
\sup _{t>0}\left(\int_{0}^{t} U(\tau) d \tau\right)^{p / q}\left(\int_{t}^{\infty} V(\tau)^{1-p^{\prime}} d \tau\right)^{p-1}<\infty
$$

is fulfilled.

Note that Theorem 2 was proved by G.Talenti, G.Tomaselli, B.Muckenhoupt [7] for $1 \leq p=q<\infty$, and by J.S.Bradley [8], V.M.Kokilashvili [9], V.G.Maz'ya [10] for $p<q$.

We say that the weight pair $\left(\omega, \omega_{1}\right)$ belongs to the class $\widetilde{A}_{p q}(\gamma), \gamma>0$, if either of the following conditions is fulfilled: a) $\omega(t)$ and $\omega_{1}(t)$ are increasing functions on $(0, \infty)$ and

$$
\sup _{t>0}\left(\int_{t}^{\infty} \omega(\tau) \tau^{-1-\gamma q / p^{\prime}} d \tau\right)^{p / q}\left(\int_{0}^{t / 2} \omega(\tau)^{1-p^{\prime}} \tau^{\gamma-1} d \tau\right)^{p-1}<\infty ;
$$

b) $\omega(t)$ and $\omega_{1}(t)$ are decreasing functions on $(0, \infty)$ and

$$
\sup _{t>0}\left(\int_{0}^{t / 2} \omega_{1}(\tau) \tau^{\gamma-1} d \tau\right)^{p / q}\left(\int_{t}^{\infty} \omega(\tau)^{1-p^{\prime}} \tau^{-1-\gamma p^{\prime} / q} d \tau\right)^{p-1}<\infty .
$$

Theorem 3. Let $1<p<\infty$ and the weight pair $\left(\omega, \omega_{1}\right) \in \widetilde{A}_{p}(Q) \equiv$ $\widetilde{A}_{p p}(Q)$. Then for $f \in L_{p}\left(H^{n}, \omega\left(|x|_{H}\right)\right)$ there exists $T f(x)$ for almost all $x \in H^{n}$ and

$$
\left.\int_{H^{n}}|T f(x)|^{p} \omega_{1}\left(|x|_{H}\right) d x \leq C \int_{H^{n}}|f(x)|^{p} \omega(|x|)_{H}\right) d x,
$$

where the constant $C$ does not depend on $f$. 
Corollary. If $\omega(t), t>0$ is increasing (decreasing) and the function $\omega(t) t^{-\beta}$ is decreasing (increasing) for some $\beta \in(0, Q(p-1))(\beta \in(-Q, 0))$, then $T$ is bounded on $L_{p}\left(H^{n}, \omega\left(|x|_{H}\right)\right)$.

Proof of Theorem 3. Let $f \in L_{p}\left(H^{n}, \omega\left(|x|_{H}\right)\right)$ and $\omega, \omega_{1}$ be positive increasing functions on $(0, \infty)$. We will prove that $T f(x)$ exists for almost all $x \in H^{n}$. We take any fixed $\tau>0$ and represent the function $f$ in the norm of the sum $f_{1}+f_{2}$, where

$$
f_{1}(x)=\left\{\begin{array}{ll}
f(x), & \text { if }|x|_{H}>\tau / 2 \\
0, & \text { if }|x|_{H} \leq \tau / 2
\end{array}, \quad f_{2}(x)=f(x)-f_{1}(x) .\right.
$$

Let $\omega(t)$ be a positive increasing function on $(0, \infty)$ and $f \in L_{p}\left(H^{n}, \omega\left(|x|_{H}\right)\right)$. Then $f_{1} \in L_{p}\left(H^{n}\right)$ and therefore $T f_{1}(x)$ exists for almost all $x \in H^{n}$. Now we will show that $T f_{2}$ converges absolutely for all $x:|x|_{H} \geq \tau$. Note that $C(K)=\sup _{x \in S_{H}}|K(x)|<\infty$. Hence

$$
\begin{gathered}
\left|T f_{2}(x)\right| \leq C(K) \int_{|y|_{H} \leq \tau / 2} \frac{|f(y)|}{\left|x y_{-1}\right|_{H}^{Q}} d y \leq \\
\leq\left(\frac{2}{\tau}\right)^{\frac{Q}{p}} \int_{|y|_{H} \leq \tau / 2} \frac{|f(y)| \omega\left(|y|_{H}\right)^{\frac{1}{p}}}{\omega\left(|y|_{H}\right)^{\frac{1}{p}}} d y,
\end{gathered}
$$

since $\left|x y^{-1}\right|_{H} \geq|x|_{H}-|y|_{H} \geq \tau / 2$. Thus, by the Hölder inequality we can estimate (2) as

$$
\left|T f_{2}(x)\right| \leq C \tau^{-Q / p}\|f\|_{L_{p}\left(H^{n}, \omega\left(|x|_{H}\right)\right)}\left(\int_{0}^{\tau / 2} \omega(t)^{1-p^{\prime}} t^{Q-1} d t\right)^{1 / p^{\prime}} .
$$

Therefore $T f_{2}(x)$ converges absolutely for all $x:|x|_{H} \geq \tau$ and thus $T f(x)$ exists for almost all $x \in H^{n}$. Assume $\bar{\omega}_{1}(t)$ to be an arbitrary continuous increasing function on $(0, \infty)$ such that $\bar{\omega}_{1}(t) \leq \omega_{1}(t), \bar{\omega}_{1}(0)=\omega_{1}(0+)$ and $\bar{\omega}_{1}(t)=\int_{0}^{t} \varphi(\tau) d \tau+\bar{\omega}_{1}(0), t \in(0, \infty)$ (it is obvious that such $\bar{\omega}_{1}(t)$ exists; for example, $\left.\bar{\omega}_{1}(t)=\int_{0}^{t} \omega_{1}^{\prime}(\tau) d \tau+\omega_{1}(t)\right)$.

We observe that the condition a) implies

$$
\exists C_{1}>0, \quad \forall t>0, \quad \omega_{1}(t) \leq C_{1} \omega(t / 2) .
$$

Indeed, from

$$
\begin{gathered}
\exists C_{2}>0, \quad \forall t>0, \\
\left(\int_{t}^{\infty} \varphi(\tau) \tau^{-Q(p-1)} d \tau\right)\left(\int_{0}^{t / 2} \omega(\tau)^{1-p^{\prime}} \tau^{Q-1} d \tau\right)^{p-1} \leq C_{2}
\end{gathered}
$$


we obtain (3), since

$$
\begin{gathered}
\int_{t}^{\infty} \omega_{1}(\tau) \tau^{-1-Q(p-1)} d \tau \geq C \omega_{1}(t) t^{-Q(p-1)} \\
\left(\int_{0}^{t / 2} \omega(\tau)^{1-p^{\prime}} \tau^{Q-1} d \tau\right)^{p-1} \leq C \omega(t / 2)^{-1} t^{Q(p-1)}
\end{gathered}
$$

and, besides,

$$
\begin{gathered}
\frac{1}{Q(p-1)} \int_{t}^{\infty} \varphi(\tau) \tau^{-Q(p-1)} d \tau=\int_{t}^{\infty} \varphi(\tau) d \tau \int_{\tau}^{\infty} \lambda^{-1-Q(p-1)} d \lambda= \\
=\int_{t}^{\infty} \lambda^{-1-Q(p-1)} d \lambda \int_{t}^{\lambda} \varphi(\tau) d \tau \leq \int_{t}^{\infty} \omega_{1}(\tau) \tau^{-1-Q(p-1)} d \tau .
\end{gathered}
$$

We have

$$
\begin{gathered}
\|T f\|_{L_{p}, \bar{\omega}_{1}\left(H^{n}\right)} \leq\left(\int_{H^{n}}|T f(x)|^{p} d x \int_{0}^{|x|_{H}} \varphi(t) d t\right)^{1 / p}+ \\
+\left(\bar{\omega}_{1}(0) \int_{H^{n}}|T f(x)|^{p} d x\right)^{1 / p}=A_{1}+A_{2} .
\end{gathered}
$$

If $\omega(0+)>0$, then $L_{p}\left(H^{n}, \omega\left(|x|_{H}\right)\right) \subset L_{p}\left(H^{n}\right)$, and if $\omega(0+)=0$, then $\bar{\omega}(t) \leq \omega_{1}(t) \leq C \omega(t / 2)$ implies $\bar{\omega}_{1}(0)=0$. Therefore in the case $\omega(0+)=0$ we have $A_{2}=0$.

If $\omega(0)>0$, then $f \in{L_{p}\left(\mathbb{R}^{n}\right)}$ and we have

$$
\begin{gathered}
A_{2} \leq C\left(\bar{\omega}_{1}(0) \int_{H^{n}}|f(x)|^{p} d x\right)^{1 / p} \leq C\left(\int_{H^{n}}|f(x)|^{p} \omega_{1}\left(|x|_{H}\right) d x\right)^{1 / p} \leq \\
\leq C\|f\|_{L_{p}\left(H^{n}, \omega\left(|x|_{H}\right)\right) .}
\end{gathered}
$$

Now we can write

$$
A_{1} \leq\left(\int_{0}^{\infty} \varphi(t) d t \int_{|x|_{H}>t} \mid T f(x)^{p} d x\right)^{1 / p} \leq A_{11}+A_{12} .
$$

where

$$
\begin{aligned}
& A_{11}^{p}=\int_{0}^{\infty} \varphi(t) d t \int_{|x|_{H}>t}\left|\int_{|y|_{H}>t / 2} K\left(x, y^{-1}\right) f(y) d y\right|^{p} d x, \\
& A_{12}^{p}=\int_{0}^{\infty} \varphi(t) d t \int_{|x|_{H}>t}\left|\int_{|y|_{H}<t / 2} K\left(x, y^{-1}\right) f(y) d y\right|^{p} d x .
\end{aligned}
$$


The relation

$$
\int_{|y|_{H}>t / 2}|f(y)|^{p} d y \leq \frac{1}{\omega(t / 2)} \int_{|y|_{H}>t / 2}|f(y)|^{p} \omega\left(|y|_{H}\right) d y
$$

implies $f \in L_{p}\left(\left\{y \in H^{n}:|y|_{H}>t\right\}\right)$ for any $t>0$.

Hence, on account of (3), we have

$$
\begin{gathered}
A_{11} \leq C\left(\int_{0}^{\infty} \varphi(t) d t \int_{|x|_{H}>t / 2}|f(x)|^{p} d x\right)^{1 / p}= \\
=C\left(\int_{H^{n}}|f(x)|^{p} d x \int_{0}^{2|x|_{H}} \varphi(t) d t\right)^{1 / p} \leq \\
\leq C\left(\int_{H^{n}}|f(x)|^{p} \omega_{1}\left(2|x|_{H}\right) d x\right)^{1 / p} \leq C\|f\|_{\left.L_{p, \omega(|x|}\right)}\left(H^{n}\right) .
\end{gathered}
$$

Obviously, if $|x|_{H}>t,|y|_{H}<t / 2$, then $\frac{1}{2}|x|_{H} \leq\left|y^{-1} x\right|_{H} \leq \frac{3}{2}|x|_{H}$. Therefore

$$
\begin{gathered}
\int_{|x|_{H}>t}\left|\int_{|y|_{H}<t / 2} K\left(x y^{-1}\right) f(y) d y\right|^{p} d x \leq \\
\left.\leq C(K) \int_{|x|_{H}>t} \int_{|y|_{H}<t / 2}\left|x y^{-1}\right|_{H}^{-Q}|f(y)| d y\right)^{p} d x \leq \\
\leq 2^{Q p} C(K) \int_{|x|_{H}>t}|x|_{H}^{-Q p} d x\left(\int_{|y|_{H}<t / 2}|f(y)| d y\right)^{p} .
\end{gathered}
$$

Taking the $H$-polar coordinates $x=\delta_{\varrho} \bar{x}, \varrho=|x|_{H}, \bar{x} \in S_{H}$ we can write

$$
\int_{|x|_{H}>t}|x|_{H}^{-Q p} d x=\int_{S_{H}} d \sigma(\bar{x}) \int_{0}^{\infty} \varrho^{Q-1-Q p} d \varrho=C t^{Q-Q p} .
$$

For $\alpha>Q\left(1+\frac{1}{p^{\prime}}\right)$, by virtue of the Hölder inequality, we have

$$
\begin{gathered}
\int_{|y|_{H}<t / 2}|f(y)| d y=\alpha \int_{S_{H}} d \sigma(\bar{y}) \int_{0}^{t / 2} \varrho^{Q-\alpha-1}\left|f\left(\delta_{\varrho} \bar{y}\right)\right| d \varrho \int_{0}^{\varrho} s^{\alpha-1} d s= \\
=\alpha \int_{0}^{t / 2} s^{\alpha-1} d s \int_{s<|y|_{H}<t / 2}|f(y)||y|_{H}^{-\alpha} d y \leq
\end{gathered}
$$




$$
\begin{gathered}
\leq \int_{0}^{t / 2} s^{\alpha-1} d s\left(\int_{s<|y|_{H}<t / 2}|f(y)|^{p}|y|_{H}^{-Q p} d y\right)^{1 / p} \times \\
\times\left(\int_{s<|y|_{H}<t / 2}|y|_{H}^{(Q-\alpha) p^{\prime}} d y\right)^{1 / p^{\prime}} \leq \\
\leq C \int_{0}^{t / 2} s^{Q+\frac{Q}{p^{\prime}}}\left(\int_{s<|y|_{H}<t / 2}|f(y)|^{p}|y|_{H}^{-Q p} d y\right)^{1 / p} d s .
\end{gathered}
$$

Consequently

$$
\begin{gathered}
A_{12} \leq C\left\{\int_{0}^{\infty} \varphi(2 t) t^{-Q(p-1)} \times\right. \\
\left.\times\left[\int_{0}^{t} s^{Q\left(1+\frac{1}{p^{\prime}}\right)}\left(\int_{|y|_{H} \geq s}|f(y)|^{p}|y|_{H}^{-Q p} d y\right)^{1 / p} d s\right]^{p} d t\right\}^{1 / p} .
\end{gathered}
$$

By (4) and Theorem 2

$$
\begin{gathered}
A_{12} \leq C\left[\int _ { 0 } ^ { \infty } s ^ { Q p ( 1 + \frac { 1 } { p ^ { \prime } } ) } \left(\int_{|y|_{H}>s}|f(y)|^{p} \times\right.\right. \\
\left.\left.\times|y|^{-Q p} d y\right) \omega(s) s^{-(Q-1)(p-1)} d s\right]^{1 / p}= \\
=C\left(\int_{0}^{\infty} s^{-1+Q p} \omega(s) d s \int_{|y|_{H}>s}|f(y)|^{p}|y|_{H}^{-Q p} d y\right)^{1 / p}= \\
=C\left(\int_{H^{n}}|f(y)|^{p}|y|_{H}^{-Q p} \int_{0}^{|y|_{H}} \omega(s) s^{-1+Q p} d s\right)^{1 / p} \leq \\
\leq C\left(\int_{H^{n}}|f(y)|^{p} \omega\left(|y|_{H}\right) d y\right)^{1 / p} .
\end{gathered}
$$

Hence we obtain (1) for $\omega_{1}(t)=\bar{\omega}_{1}(t)$. Now, by the Fatou theorem, the inequality (1) is fulfilled.

Theorem 3 was earlier announced in [11].

A similar reasoning can be used to prove the analogue of Theorem 3 for the operator $T_{\alpha}: f \rightarrow T_{\alpha} f$ where

$$
T_{\alpha} f(x)=\int_{H^{n}}\left|x y^{-1}\right|_{H}^{\alpha-Q} f(y) d y, \quad 0<\alpha<Q .
$$

Namely, we have 
Theorem 4. Let $0<\alpha<Q, 1<p<\frac{Q}{\alpha}, \frac{1}{p}-\frac{1}{q}=\frac{\alpha}{Q}$ and the weights $\left(\omega, \omega_{1}\right)$ be monotone positive functions on $(0, \infty)$. Then the inequality

$$
\left(\int_{H^{n}}\left|T_{\alpha} f(x)\right|^{q} \omega_{1}\left(|x|_{H}\right) d x\right)^{1 / q} \leq C\left(\int_{H^{n}}|f(x)|^{p} \omega\left(|x|_{H}\right) d x\right)^{1 / p}
$$

holds if and only if $\left(\omega, \omega_{1}\right) \in \widetilde{A}_{p, q}(Q)$.

Remark. In the case of a homogeneous group the analogue of Theorem 4 is also valid (see [12]).

For monotone weights one can find the weighted $L_{p}$-estimates for a Calderon-Zygmund operator in [13] and [14], and for the anisotropic case in [15].

As known [16], if $f \in C_{0}^{\infty}\left(H^{n}\right)$, then the function

$$
g(x)=C_{n} \int_{H^{n}}\left|x y^{1}\right|_{H}^{-2 n} f(y) d y
$$

is a solution of the equation $L_{0} g=f$, where $L_{0}=-\sum_{i=1}^{2 n} X_{j}^{2}$. In particular, our results lead to

Theorem 5. Let $1<p<\infty,\left(\omega, \omega_{1}\right) \in \widetilde{A}(Q), f \in L_{p}\left(H^{n}, \omega\left(|x|_{H}\right)\right)$, and $L_{0}(g)=f$. Then

$$
\begin{gathered}
\left\|X_{0} g\right\|_{L_{p}\left(H^{n}, \omega_{1}\left(|x|_{H}\right)\right)} \leq c\|f\|_{L_{p}\left(H^{n}, \omega\left(|x|_{H}\right)\right)}, \\
\left\|X_{i} X_{j} g\right\|_{\left.L_{p}\left(H^{n}, \omega_{1}(|x|)_{H}\right)\right)} \leq C\|f\|_{L_{p}\left(H^{n}, \omega\left(|x|_{H}\right)\right)}, \\
i, j=1,2, \ldots, 2 n .
\end{gathered}
$$

Theorem 6. Let $1<p<q<\infty, \frac{1}{p}-\frac{1}{q}=\frac{1}{Q},\left(\omega, \omega_{1}\right) \in \widetilde{A}_{p q}(Q)$, $f \in L_{p}\left(H^{n}, \omega\left(|x|_{H}\right)\right)$, and $L_{0} g=f$. Then

$$
\left\|X_{i} g\right\|_{L_{q}\left(H^{n}, \omega_{1}\left(|x|_{H}\right)\right)} \leq C\|f\|_{L_{p}\left(H^{n}, \omega\left(|x|_{H}\right)\right)}, \quad i=1,2, \ldots, 2 n .
$$

\section{REFERENCES}

1. G.B. Folland and E.M. Stein, Hardy spaces on homogeneous groups. Math. Notes, No.28, Princeton Univ. Press, 1982.

2. N.E. Taylor, Noncommutative harmonic analysis. Amer. Math. Soc., Providence, 1986.

3. A. Koranyi and S. Vagi, Singular integrals in homogeneous spaces and some problems of classical analysis. Ann. Scuola Norm. Sup. Pisa 25(1971), 575-648. 
4. R.R. Coifman and G. Weiss, Analyse harmonique noncommutative sur certains espaces homogenes. Lecture Notes in Math., v. 242, Springer, Berlin etc., 1971.

5. J.R. Dorronsoro, Weighted Hardy spaces on Siegel's half-planes. Math. Nachr. 125(1986), 103-119.

6. R.R. Coifman and C. Fefferman, Weighted norm inequalities for maximal functions and singular integrals. Studia Math. 51(1974), 241-250.

7. B. Muckenhoupt, Hardy's inequality with weights. Studia Math. 44(1972), No.1, 31-38.

8. J.S. Bradley, Hardy inequalities with mixed norms. Can. Math. Bull. 21(1978), No. 4, 405-408.

9. V.M. Kokilashvili, On Hardy inequalities in weighted spaces (Russian). Bull. Acad. Sci. Georgian SSR 96(1979), No.2, 37-40.

10. V.G. Maz'ya, S.L. Sobolev spaces. Leningrad University Press, Leningrad, 1985.

11. V.S. Guliev, On the boundedness of singular operators on the Heisenberg group in weighted generalized Hölder spaces and weighted $L_{p}$-spaces. (Russian) Dokl. Akad. Nauk SSSR 316(1991), No.2, 274-278.

12. V.S. Guliev, Two-weighted $L_{p}$-estimates for some operators on homogeneous Lie groups. (Russian) Current problems of the theory of functions (Russian). Abstracts of All-Union School-Conference, 33-34, Baku, 1985.

13. S.K. Abdulaev, On some classes of integral operators in spaces of summable functions (Russian). Dokl. Akad. Nauk SSSR 283(1985), No. 4, 777-780.

14. E.G. Guseinov, Singular integrals in the spaces of functions summable with monotone weight. (Russian) Mat. Sb. 132(174)(1977), No. 1, $28-44$.

15. V.S. Guliev, On a certain class of anisotropic integral operators and weight embedding theorems (Russian). Dokl. Akad. Nauk SSSR 304(1989), No. 6, 1289-1293; English translation: Soviet Math. Dokl. 39(1989), No. 1, 199-204.

16. G.B. Folland. A fundamental solution for a subelliptic operator. Bull. Amer. Math. Soc. 79(1973), No.2, 373-376.

(Received 15.02.1993)

Author's address:

Faculty of Mechanics and Mathematics

Baku State University

23 Lumumba St., Baku 370073

Azerbaijan 